\title{
STUDY OF WORKING - WOMEN EMPOWERMENT IN SOUTH ASIA: CASE OF PAKISTAN
}

\author{
*Farah Naseer, Lecturer Department of Sociology, Sardar Bahadur Khan Women \\ University, Quetta- Pakistan (farahnaseersoc@gmail.com) \\ ** Yasmin Syed, Lecturer, Department of Sociology, Sardar Bahadur Khan Women \\ University, Quetta- Pakistan (yasmin.syed09@gmail.com) \\ *** Safia Habib, Lecturer, Department of Sociology, Sardar Bahadur Khan Women \\ University, Quetta- Pakistan (safiashah123@gmail.com)
}

\begin{abstract}
Women are the integral part of all societies no society can progress without the active participation of women. The involvement of women in decision making process is very important in every field of life. Like many other developing countries Pakistan has also patriarchal social set up where women take decisions under the dominance of their father, husband. Objective of the study was find out the factors influencing the decision-making power of women in family matters' sample of 120 married universities teachers was selected from the three selected universities of Quetta city. Chi square analysis is used to see the influence of age and personal income on women decision making power. The result reveals that there is significant association between different basic scale and right to take decision as a wife $(p<0.020)$ or as a mother $(p<0.026)$, Married university teachers believe in husband seniority in age $(r=0.111 ; p<0.002)$ with their decision-making power. Furthermore, they achieve socio economic status $(p=<0.049)$ due to their personal income and their income play supportive role $(p=<0.014)$ in decision making process. $75 \%$ respondents free to spend their income on household expenditure $(p=<0.026)$ but have less authority over property related decisions. Overall women education, age, occupation and income has a significant effect on household decision making.
\end{abstract}

KEYWORDS: Patriarchal Society, Decision Making Power. Age, Education, Income

\section{INTRODUCTION}

Women play very significant role for the welfare of their family. Multiple task is performed by them as wife, mother, as homemaker. Hence 


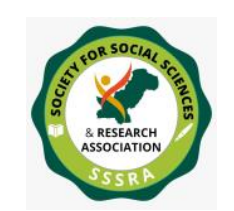

Pak. Journal of Int'L Affairs, Vol 1, Issue 2 (2018)

Study of Working - Women ,...

the decision-making power of working women in family matters has been observed as one of the crucial factor which may affect the welfare of their family (Safilois, 1983). To achieve the equity and peace with in the family and country the active participation of women in all type of decisions is very important(Mahmooda,2008). women decision making power in family matters in patriarchal and male dominant societies like Pakistan they have very limited access and power to take decision about family matters even they are not allowed to take decisions to decide for their own life. Pertaining to the above statement (Taj, et al 2004) stated in south Asian countries like Pakistan the status of women is lower than male counterpart, she plays very little part in family decisions.

The repot of (Unicef 2006) revealed that in south Asia, Pakistan is the country with the widest gender gap and discrimination, against women continuous to persist in all walks of life that women are compelled to live according to their cultural and traditional norms. When we try to view the picture of female in the scenario of Pakistan a very deplorable state of women revolves around our eyes because they are shown to be very miserable, pathetic, submissive, humble and so on they are considered to be less knowledge able and talented whose main task to perform only house hold chores, child rearing( Rizvi,P,1980). It is also observed that decision making power is not only influenced by cultural setting (Rodman,1972) like other patriarchal societies, in Pakistani society men authority in family decision is observed simply by virtue of being men and head of family( Schuler et al, 1996;Sultana 2010).

\section{STATUS OF WOMEN IN SOUTH ASIA}

In Indian constitution women can enjoy equal status with men but to achieve the present position she suffered a lot, firstly they faced gender inequality in every sphere of life secondly Indian women depends on their male family members in every matters of their lives thirdly they are not allowed to speak loudly in front of their elder family members of their inlaws Fourthly in case of widowhood she became more dependent on their male members even they are not allowed to attend many social family ceremonies as well as they have little participation in social, economic and political life of society.

The policy makers recognized the equal social position of women along with men after independency of India. Women empowerment means to prepare a woman to be self -reliant, economically strong and enable them to face and kind of tough situation and plays their positive role in 
social development activities also participate actively in decision making process. In present scenario in India women became able to achieve respectable status/ position in all fields of life but still she is culturally restricted and face gender discrimination of society( ). That current situation proclaims that women role in family decision is not up to the mark. The key factor is patriarchal family system, male dominancy and narrow perception of male genders towards women talent and expertise.

\section{STATEMENT OF THE PROBLEM}

Researcher has observed that there is a wide gulf between the ideology and practice. Many debates and speeches support women in terms of their importance and vitality in various spheres of life but little success has been achieved in practical scenario. She noticed that there are various factors that create hurdles in the way of female autonomy like patriarchal system, male dominancy, influence of traditional and cultural boundaries, un accessibility towards resources and the like. Moreover, researcher stated that these above-mentioned issues are affecting the status of women in the modern era. Consequently, she is not playing her key role in the process of decision making. so, it is an hour of need to recognize women talent and expertise and give them an opportunity to work for wellbeing of the family and society.

\section{SCOPE OF THE STUDY}

Researcher expects that this current study will be helpful at national as well international level. it will be guide line for the awareness and reorganization of female vital role in family decision making process that how age, and income empower women to take decisions regarding their family matters

\section{PURPOSE OF THE STUDY}

The researcher main aim of conducting this study is to explore the importance of women in decision making process because it has been noted that female involvement in family decision not only enable them to work for their own psychological and physical health but also make them capable to work for the progress and development of their family in a better way.

\section{LITERATURE REVIEW}




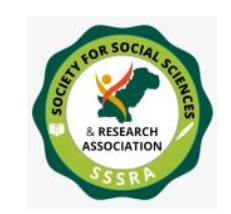

Pak. Journal of Int'L Affairs, Vol 1, Issue 2 (2018)

Study of Working - Women ,...

Education plays very important role in decision making process(Muzamil,2008). The present study claimed that decision making power of married working women is significantly related with their educational level, income and their socio-economic status. Resource theory of Blood Wolf 1960 indicate that decision making power of husband and wife highly influenced by the resources, resources refer to the couple's qualification, occupation and income. This theory also provides facts that women say "Yes" may increase in house hold when her resources are increased. Many other theories also support the Blood Wolf theory that in family if a person either men or women has more resources will have more decision-making power (Saffilios-Roths child,1969; Lamouse,1969; Lupri,1969; Kandel and Lesser 1972).

(Green, R.T ; Cunningham ,1975) compare family decision making patterns ,he observed differences between contemporary and traditional families especially within income category and age.( Hakim,A and Aziz ,A,1998) status of women in Pakistan is affect by the socio cultural restrictions, customs and exercise of patriarchy, despite these due to education and employment of women little change has been observed in the decision making at household level.( Dutta, M, 2002) trend of paid employment among educated women is the major factor due to this transfer in authority patterns and change in husband traditional roles has been observed that increased the female decision making power at house hold level. Still major decisions are taken by husbands. (Lee,C.K ; Beatty,S.E 2002) focused on how family structure influence the decision making process. Researcher observed that wives occupational grade and their financial contribution for their families have significant influence and this influence is observed by the adolescents who are depends in their mother's occupational grade. (Khanam, R,2003) husband of working women mutually take decisions about family matters while husband of non-working wives is highly involved in family decisions. Wives education and their employment plays a significant role in their involvement in decision making about family matters. Although the exercise of gender ideology matters a lot in the husband's involvement in family decision making. (Jan,M ; Akhter ,s, 2008)the women who reliant on their husband have low decision making power.( Carlsson, F; Martinsson, P; Qin, P;Sutter,M,2009) wives highly financial contribution in family improve the standard of family relatively but in joint decisions mostly wives under the dominance of her husband decisions. (Jabeen, N; Iqbal.M.Z, 2010) conclude that women age and their duration of marriage life are the key factors that may increase the women decision making 


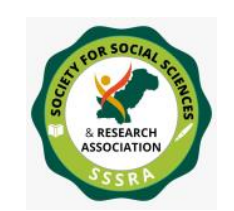

Pak. Journal of Int'L Affairs, Vol 1, Issue 2 (2018)

Study of Working - Women ,...

power at family level. (Hammad, M, 2010) examine the influence of age , income, total number of family religion on the decision making power of working women at house, economic independence and their economic contribution in family budget became the women more sovereign at family level but the researcher observed the negative association between the age of women and their involvement in decision making process about family matters, the involvement of Christian women in family matters is highly observed as compare to the Muslin women.( Arooj,S; Hussain 2013) decision making power of women influenced by the different factors like women age, nature of job, monthly income, residence, family structure, researcher conclude that women with the age of 30 and above has authority to take decision about family planning if she lives in nuclear family set up can spend their own income independently furthermore it is also observed that women having jobs in government sector had enjoy the more autonomy of decision making as compare to the women doing job in non-government sector.( Fatima ,D,2013)found the cause and effect relationship between the employment and empowerment, study reveals the significant influence of women education and their employment on their decision making power but not all types of decisions their financial independence empower the women in expenditure related decisions but still they have no authority to take decisions related family planning.

\section{OBJECTIVE}

1. To identify the factors influencing the universities married teacher's decision-making power in their family matters

\section{HYPOTHESIS}

1. Decision making power of universities married teachers in family matters is likely to be related with their age

2. Decision making power of universities married teachers in family matters is likely to be related with their personal income.

\section{RESEARCH METHODOLOGY}

Survey research method was used in the present study to collect information about large population by using the technique of structured questionnaire (Black storm\& Hursh ,1963). Quantitative research design is occupied by the researcher to analyzed the decision making power of married working women, quantitative research design provide 
immediate statistical analysis about the phenomena may also help full to test the hypothesis

\section{UNIVERSE / SAMPLE}

Quetta is multi ethical city of Baluchistan where women were varied in term of their cultural aspects. The researcher conduct study in the three purposively selected universities of Quetta city, that was Sardar bahadur khan women university, Baluchistan university of information and technology, Engineering and management science university of Baluchistan. A sample of 120 married married women were selected from these universities by using the technique of simple random sampling.

\section{TOOL OF DATA COLLECTION}

A structured questionnaire was utilized to collect the data from participants regarding decision making power that was consisted on two sections the first section related to the demographic information and the second was having the questions related to the factors like women age and their income that how they influence the decision-making power of married working women in family matters.

\section{DATA ANALYSIS}

Researcher analyzed the data by using SPSS software version 20 . Both descriptive and inferential Chi square statistics were used for presenting the influence of age and personal income on their family related matters.

\section{RESULT AND DISCUSSION}

RESPONDENTS DEMOGRAPHIC PROFILES ON THE BASIS OF AGE GROUP

\begin{tabular}{|c|c|c|c|c|c|}
\hline $\begin{array}{l}\text { Respondent's } \\
\text { characteristi } \\
\text { cs }\end{array}$ & $\begin{array}{l}\mathrm{N}=29 \\
30-34 \mathrm{n}= \\
(\%)\end{array}$ & $\begin{array}{l}\mathrm{N}=34 \\
35-39 \mathrm{n}= \\
(\%)\end{array}$ & $\begin{array}{l}\mathrm{N}=21 \\
40-44 \mathrm{n}= \\
(\%)\end{array}$ & $\begin{array}{l}\mathrm{N}=18 \\
45-49 \mathrm{n}= \\
(\%)\end{array}$ & $\begin{array}{l}\mathrm{N}=18 \\
50 \quad \& \\
\text { above } \mathrm{n}= \\
(\%)\end{array}$ \\
\hline \multicolumn{6}{|c|}{ Basic pay scale of respondent } \\
\hline BPS 18 & $26(42.6 \%$ & $\begin{array}{l}22(36.1 \% \\
)\end{array}$ & $8(13.1 \%)$ & $3(4.9 \%)$ & $2(3.3 \%)$ \\
\hline BPS 19 & $3(10.0 \%)$ & $12(40.0 \%$ & $9(30.0 \%)$ & $5(16.7 \%)$ & $1(3.3 \%)$ \\
\hline
\end{tabular}


Pak. Journal of Int'L Affairs, Vol 1, Issue 2 (2018)

Study of Working - Women ,...

\begin{tabular}{|c|c|c|c|c|c|}
\hline BPS 20 & $0(0.0 \%)$ & $0(0.0 \%)$ & $4(23.5 \%)$ & $5(29.4 \%)$ & $8(47.8 \%)$ \\
\hline BPS 21 & $0(0.0 \%)$ & $0(0.0 \%)$ & $0(0.0 \%)$ & $5(41.7 \%)$ & $7(58.3 \%)$ \\
\hline \multicolumn{6}{|c|}{ Respondent educational qualification } \\
\hline B. S & $5(62.5 \%)$ & $3(37.5 \%)$ & $0(0.0 \%)$ & $0(0.0 \%)$ & $0(0.0 \%)$ \\
\hline Master's & $8(28.6 \%)$ & $11(39.3 \%$ & $1(3.6 \%)$ & $5(17.9 \%)$ & $3(10.7 \%)$ \\
\hline M.phil & $15(31.9 \%$ & $17(36.2 \%$ & $7(14.9 \%)$ & $4(8.5 \%)$ & $4(8.5 \%)$ \\
\hline Phd & $1(2.9 \%)$ & $3(8.8 \%)$ & $13(38.2 \%$ & $9(26.5 \%)$ & $8(23.5 \%)$ \\
\hline Post doctorate & $0(0.0 \%)$ & $0(0.0 \%)$ & $0(0.0 \%)$ & $0(0.0 \%)$ & $3(100.0 \%$ \\
\hline \multicolumn{6}{|c|}{ Respondent job experience } \\
\hline $5-9$ & $\begin{array}{l}24(49.0 \% \\
)\end{array}$ & $17(34.7 \%$ & $5(10.2 \%)$ & $3(6.1 \%)$ & $0(0.0 \%)$ \\
\hline $10-14$ & $4(14.3 \%)$ & $14(50.0 \%$ & $6(21.4 \%)$ & $2(7.1 \%)$ & $2(7.1 \%)$ \\
\hline $15-19$ & $1(8.3 \%)$ & $1(8.3 \%)$ & $5(41.7 \%)$ & $5(41.7 \%)$ & $0(0.0 \%)$ \\
\hline $20-24$ & $0(0.0 \%)$ & $2(40.0 \%)$ & $1(20.0 \%)$ & $1(20.0 \%)$ & $1(20.0 \%)$ \\
\hline $25-29$ & $0(0.0 \%)$ & $0(0.0 \%)$ & $4(38.8 \%)$ & $2(15.4 \%)$ & $7(53.8 \%)$ \\
\hline $30 \&$ above & $0(0.0 \%)$ & $0(0.0 \%)$ & $0(0.0 \%)$ & $5(38.5 \%)$ & $8(61.5 \%)$ \\
\hline \multicolumn{6}{|c|}{ Respondent settlement pattern } \\
\hline Domicile & $9(16.4)$ & $11(20.0 \%$ & $14(25.5 \%$ & $9(16.4 \%)$ & $12(21.8 \%$ \\
\hline Local & $20(32.3)$ & $\begin{array}{l}23(37.1 \% \\
)\end{array}$ & $7(11.3 \%)$ & $6(9.7 \%)$ & $6(9.7 \%)$ \\
\hline KPK & $0(0.0 \%)$ & $0(0.0 \%)$ & $0(0.0 \%)$ & $\begin{array}{l}3(100.0 \% \\
)\end{array}$ & $0(0.0 \%)$ \\
\hline \multicolumn{6}{|c|}{ Residential pattern } \\
\hline Rural & $9(26.5 \%)$ & $12(35.3 \%$ & $5(14.7 \%)$ & $3(8.8 \%)$ & $5(14.7 \%)$ \\
\hline Urban & $20(23.3 \%$ & $22(25.6 \%$ & $16(18.6 \%$ & $15(17.4 \%$ & $13(15.1 \%$ \\
\hline \multicolumn{6}{|c|}{ Type of marriage } \\
\hline Love & $5(26.3 \%)$ & $5(26.3 \%)$ & $6(31.6 \%)$ & $2(10.5 \%)$ & $1(5.3 \%)$ \\
\hline Arrange & $\begin{array}{l}24(23.8 \% \\
)\end{array}$ & $\begin{array}{l}29(28.7 \% \\
)\end{array}$ & $\begin{array}{l}15(14.9 \% \\
)\end{array}$ & $\begin{array}{l}16(15.8 \% \\
\end{array}$ & $\begin{array}{l}17(16.8 \% \\
\end{array}$ \\
\hline \multicolumn{6}{|c|}{ Type of marriage } \\
\hline Exogamy & $5(26.3 \%)$ & $5(26.3 \%)$ & $6(31.6 \%)$ & $2(10.5 \%)$ & $1(5.3 \%)$ \\
\hline Endogamy & $24(23.8 \%$ & $29(28.7 \%$ & $15(14.9 \%$ & $16(15.8 \%$ & $17(16.8 \%$ \\
\hline
\end{tabular}


Pak. Journal of Int'L Affairs, Vol 1, Issue 2 (2018)

Study of Working - Women ,...

\begin{tabular}{|c|c|c|c|c|c|}
\hline & ) & ) & ) & ) & ) \\
\hline \multicolumn{6}{|c|}{ Respondent husband's qualification } \\
\hline $\begin{array}{l}\text { Under } \\
\text { graduation }\end{array}$ & $2(16.7 \%)$ & $8(66.7 \%)$ & $1(8.3 \%)$ & $1(8.3 \%)$ & $0(0.0 \%)$ \\
\hline Graduation & $\begin{array}{l}20(27.0 \% \\
\end{array}$ & $\begin{array}{l}22(29.7 \% \\
)\end{array}$ & $\begin{array}{l}15(20.3 \% \\
)\end{array}$ & $8(10.8 \%)$ & $9(12.2 \%)$ \\
\hline M.phil & $6(66.7 \%)$ & $2(22.7 \%)$ & $0(0.0 \%)$ & $1(11.1 \%)$ & $0(0.0 \%)$ \\
\hline Phd & $0(0.0 \%)$ & $0(0.0 \%)$ & $2(16.7 \%)$ & $7(58.3 \%)$ & $3(25.2 \%)$ \\
\hline Post graduate & $1(7.7 \%)$ & $2(15.4 \%)$ & $3(23.1 \%)$ & $1(7.7 \%)$ & $6(46.2 \%)$ \\
\hline \multicolumn{6}{|c|}{ Family monthly income } \\
\hline $\begin{array}{l}10,0000- \\
20,0000\end{array}$ & $\begin{array}{l}15(51.7 \% \\
\end{array}$ & $\begin{array}{l}10(34.5 \% \\
\end{array}$ & $3(10.3 \%)$ & $1(3.4 \%)$ & $0(0.0 \%)$ \\
\hline $\begin{array}{l}20,0000- \\
30,0000\end{array}$ & $10(25.0 \%$ & $14(35.0 \%$ & $8(20.0 \%)$ & $2(5.0 \%)$ & $6(15.0 \%)$ \\
\hline $\begin{array}{l}40,0000 \quad \& \\
\text { above }\end{array}$ & $4(7.8 \%)$ & $10(19.6 \%$ & $10(19.6 \%$ & $\begin{array}{l}15(29.4 \% \\
)\end{array}$ & $\begin{array}{l}12(23.5 \% \\
)\end{array}$ \\
\hline \multicolumn{6}{|c|}{ Who takes decision in respondent's family } \\
\hline Elder male & $13(35.1 \%$ & $19(51.4 \%$ & $1(2.7 \%)$ & $3(18.1 \%)$ & $1(2.7 \%)$ \\
\hline Elder female & $6(85.7 \%)$ & $0(0.0 \%)$ & $1(14.3 \%)$ & $0(0.0 \%)$ & $0(0.0 \%)$ \\
\hline Mutually & $3(7.0 \%)$ & $6(14.0 \%)$ & $\begin{array}{l}15(34.9 \% \\
)\end{array}$ & $\begin{array}{l}10(23.3 \% \\
)\end{array}$ & $9(20.9 \%)$ \\
\hline $\mathrm{Me}$ & $1(14.3 \%)$ & $2(28.6 \%)$ & $1(14.3 \%)$ & $0(0.0 \%)$ & $3(42.9 \%)$ \\
\hline My husband & $6(23.1 \%)$ & $7(26.9 \%)$ & $3(11.5 \%)$ & $5(19.2 \%)$ & $5(19.2 \%)$ \\
\hline
\end{tabular}

\section{HYPOTHESIS NO 1}

DECESION MAKING POWER OF MARRIED UNIVERSIY

TEACHERS IN FAMILY MATTERS IS LIKELY TO BE RELATED

WITH THEIR AGE

\begin{tabular}{|l|c|c|c|c|c|c|c|}
\hline $\begin{array}{c}\text { Respondent } \\
\text { Basic } \\
\text { Pay Scale }\end{array}$ & Agree & Undecided & Disagree & $\begin{array}{c}\text { Chi- } \\
\text { square }\end{array}$ & $\begin{array}{c}\text { df } \\
\text { p- } \\
\text { value }\end{array}$ & $\begin{array}{c}\text { R- } \\
\text { value }\end{array}$ \\
\hline \multicolumn{7}{|c|}{ Age factor has an impact on decision } \\
\cline { 1 - 4 } BPS 18 & $54(90.0)$ & $2(3.3)$ & $4(6.7)$ & & & & \\
\hline BPS 19 & $18(64.3)$ & $0(0.0)$ & 10 & & & \\
\hline
\end{tabular}


Pak. Journal of Int'L Affairs, Vol 1, Issue 2 (2018)

Study of Working - Women ,...

\begin{tabular}{|c|c|c|c|c|c|c|}
\hline & & & $(35.7)$ & & & \multirow[t]{4}{*}{0.001} \\
\hline BPS 20 & $14(73.7)$ & $2(10.5)$ & $3(15.8)$ & 22.064 & \multirow[t]{3}{*}{6} & \\
\hline BPS 21 & $8(61.5)$ & $3(23.1)$ & $2(15.4)$ & & & \\
\hline Total & $94(78.3)$ & $7(5.8)$ & $\begin{array}{c}19 \\
(15.8)\end{array}$ & & & \\
\hline \multicolumn{7}{|c|}{ Right to take decision as a wife } \\
\hline BPS 18 & $51(85.0)$ & $3(5.0)$ & $6(10.0)$ & & & \multirow{5}{*}{0.020} \\
\hline BPS 19 & $18(64.3)$ & $7(25.0)$ & $3(10.7)$ & & & \\
\hline BPS 20 & $11(57.9)$ & $7(36.8)$ & $1(5.3)$ & 15.016 & 6 & \\
\hline BPS 21 & $11(84.6)$ & $2(15.4)$ & $0(0.0)$ & & & \\
\hline Total & $91(75.8)$ & $19(15.8)$ & $10(8.3)$ & & & \\
\hline
\end{tabular}

Right to take decision as a mother

\begin{tabular}{|c|c|c|c|c|c|c|c|}
\hline BPS 18 & $52(86.7)$ & $1(1.7)$ & $7(11.7)$ & \multirow{5}{*}{14.336} & \multirow{5}{*}{6} & \multirow{5}{*}{0.026} & \\
\hline BPS 19 & $23(82.1)$ & $5(17.9)$ & $0(0.0)$ & & & & \\
\hline BPS 20 & $17(89.5)$ & $2(10.5)$ & $0(0.0)$ & & & & \\
\hline BPS 21 & $12(92.3)$ & $0(0.0)$ & $1(7.7)$ & & & & \\
\hline Total & $104(86.7)$ & $8(6.7)$ & $8(6.7)$ & & & & \\
\hline & & niority o & usband a & in taki & de & sions & \\
\hline BPS 18 & $45(75.0)$ & $2(3.3)$ & $13(21.7)$ & & & & \\
\hline BPS 19 & $16(57.1)$ & $2(7.1)$ & $10(35.7)$ & & & & \\
\hline BPS 20 & $9(47.4)$ & $7(36.8)$ & $3(15.8)$ & 20.683 & 6 & 0.002 & .111 \\
\hline BPS 21 & $8(61.5)$ & $3(23.1)$ & $2(15.4)$ & & & & \\
\hline Total & $78(65.0)$ & $14(11.7)$ & $28(23.3)$ & & & & \\
\hline
\end{tabular}

\section{RESULT}

$90.0 \%$ respondents in the group of BPS 18 agreed that age has an impact on decisions regarding Family matters. $73.7 \%, 64.3 \%, 61.5 \%$, in the group of BPS 20, BPS 19, BPS 21, respectively also show willingness. It shows significant association $($ Chi- square $=22.064 ; \mathrm{df}=6 ; \mathrm{p}=0.001$ ) among different pay scales. Decision making power of women is positively correlated $(r=.210)$ with the age. Making decisions about family matters as a wife is significantly associated (Chi- square $=15.016 ; \mathrm{df}=6$; $\mathrm{p}=0.020$ ) with the basic pay scale (BPS) $85.0 \%, 84.6 \%$, respondents belonged to BPS 18 and BPS 21 respectively have much authority to take 
decision as a wife about their family matters where as $64.3 \%$ from BPS 19 and $57.9 \%$ from BPS 20 agreed that they have a right to make decisions about family matters. Result reveals that there is significant association (Chi-square $=14.336 ; \mathrm{df}=6 ; \mathrm{p}=0.026$ ) between different basic pay scales and the right to take decision as a mother about family matters. 92.3\% respondents who belong to BPS 21 possess much authority. $89.5 \%, 86.7 \%$, $82.1 \%$ in the group of BPS 20, BPS 18 , BPS 19 respectively have a right to rake decision as a mother. A huge majority of respondents $75.0 \%$ possess that their husband can take batter decision about family matters because of seniority in age. $61.5 \%$ in BPS $21,57.1 \%$ in BPS 19 , and $47.4 \%$ in the group of BPS 21 also agreed that their husband can take batter decision about family matters. It shows significant association (Chisquare $=20.683 ; \quad d f=6 ; p=0.002$ ) among different pay scales. Married university teachers believe about male seniority in age is positively correlated $(\mathrm{r}=.111)$ with their decision-making power.

HYPOTHESIS NO. 2:

DECESION MAKING POWER OF MARRIED UNIVERSIY

TEACHERS IN FAMILY MATTERS IS LIKELY TO BE RELATED WITH THEIR PERSONAL INCOME

ROLE OF WOMEN'S INCOME IN DECISION MAKING

\begin{tabular}{|c|c|c|c|c|c|c|}
\hline $\begin{array}{l}\text { Responden } \\
\text { t basic pay } \\
\text { scale }\end{array}$ & Agree & $\begin{array}{l}\text { Undecide } \\
\text { d }\end{array}$ & Disagree & $\begin{array}{c}\text { Chi- } \\
\text { square }\end{array}$ & df & $\begin{array}{l}\text { P- } \\
\text { value }\end{array}$ \\
\hline \multicolumn{7}{|c|}{ Role of income in decision making } \\
\hline BPS 18 & $\begin{array}{c}34 \\
(56.7)\end{array}$ & $4(6.7)$ & $22(36.7)$ & & & \multirow{5}{*}{$\begin{array}{r}0.01 \\
4\end{array}$} \\
\hline BPS 19 & $\begin{array}{c}23 \\
(82.1)\end{array}$ & $2(7.1)$ & $3(10.7)$ & & & \\
\hline BPS 20 & $\begin{array}{c}12 \\
(63.2)\end{array}$ & $3(15.8)$ & $4(21.1)$ & $\begin{array}{c}15.92 \\
9\end{array}$ & 6 & \\
\hline BPS 21 & $\begin{array}{c}13(10 \\
0.0)\end{array}$ & $0(0.0)$ & $0(0.0)$ & & & \\
\hline Total & $\begin{array}{c}82 \\
(68.3) \\
\end{array}$ & $9(7.5)$ & $29(24.2)$ & & & \\
\hline
\end{tabular}




\section{Spending independent economic life}

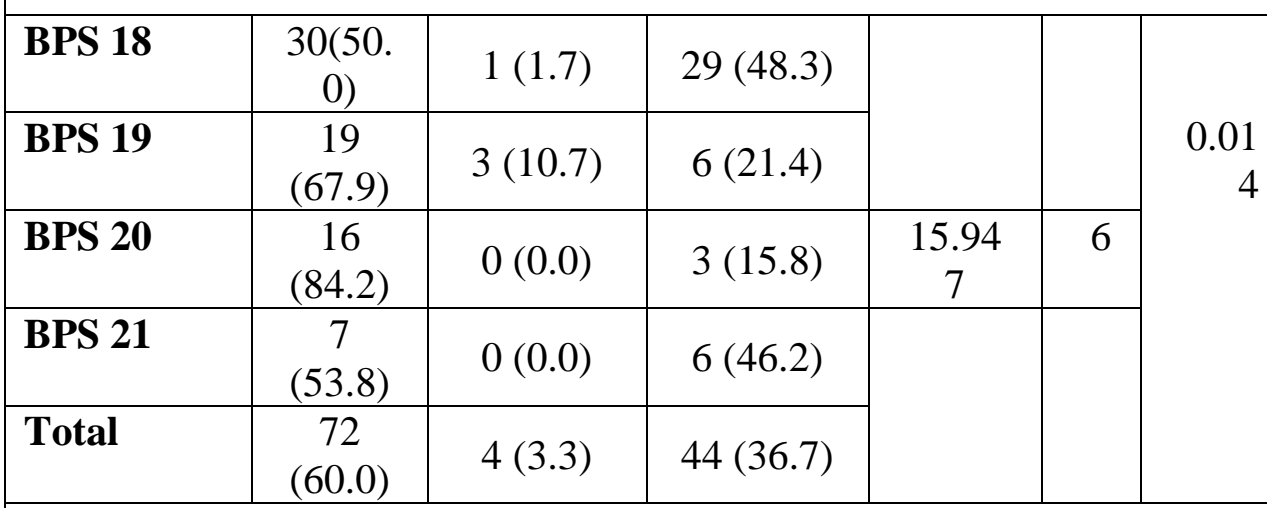

Achieve successful social economic status

\begin{tabular}{|l|c|c|c|c|r|r|}
\hline BPS 18 & $\begin{array}{c}52 \\
(86.7)\end{array}$ & $2(3.3)$ & $6(10.0)$ & & & \\
\cline { 1 - 4 } BPS 19 & $\begin{array}{c}24 \\
(85.7)\end{array}$ & $2(7.1)$ & $2(7.1)$ & & 0.04 \\
BPS 20 & $\begin{array}{c}18 \\
(94.7)\end{array}$ & $0(0.0)$ & $1(5.3)$ & $\begin{array}{c}12.66 \\
3\end{array}$ & 6 & \\
\cline { 1 - 4 } BPS 21 & $\begin{array}{c}8 \\
(61.5)\end{array}$ & $0(0.0)$ & $5(38.5)$ & & & \\
\cline { 1 - 4 } Total & $\begin{array}{c}102 \\
(85.0)\end{array}$ & $4(3.3)$ & $14(11.7)$ & & & \\
\hline
\end{tabular}

\section{RESULT}

All the respondents (100.0\%) in the category of BPS 21 and $82.1 \%$ (23/28) in BPS 19 respondents accept that their income play strong role to make them independent in decision making regarding family matters while $63.2 \%(12 / 19)$ of BPS 20 and 56.7\% (34/60) of BPS 18 respondents also agreed that their income play major role to make them empowered. Decision making power is significantly associated with respondents' income (Chi-square $=15.929 ; \mathrm{df}=6 ; \mathrm{p}=0.014$ ). Majority of the respondents $84.2 \%$ from the BPS 20, 67.9\% from the BPS 19, 53.8\% from the BPS 21 and $50.0 \%$ from the BPS 18 respectively enjoyed the independent economic life. It is significantly associated with the basic pay scale of respondents' (Chi-square $=15.947 ; \mathrm{df}=6 ; \mathrm{p}=0.014)$. The chi-square value 
shows significant association between basic pay scale and social economic status of respondent (Chi-square $=12.663 ; \mathrm{df}=6 ; \mathrm{p}=0.049$ ). 94.7\%, 86.7\%, $85.7 \%$.and $61.5 \%$ from the group of BPS 20, BPS 18, BPS 19 and BPS 21 respectively agreed that they gain successful social status. It has correlated $(r=.138)$ with income of married employed women of university.

\section{LESS POWER OF WOMEN PROPERTY RELATED DECISIONS}

\begin{tabular}{|c|c|c|c|c|c|c|l|}
\hline $\begin{array}{c}\text { Responde } \\
\text { nt basic } \\
\text { pay scale }\end{array}$ & Agree & $\begin{array}{c}\text { Undecide } \\
\text { d }\end{array}$ & $\begin{array}{c}\text { Disagre } \\
\text { e }\end{array}$ & $\begin{array}{c}\text { Chi- } \\
\text { squar } \\
\text { e }\end{array}$ & $\begin{array}{c}\text { d } \\
\text { f }\end{array}$ & $\begin{array}{c}\text { p- } \\
\text { valu } \\
\text { e }\end{array}$ & $\begin{array}{l}\text { valu } \\
\text { ealu }\end{array}$ \\
\hline
\end{tabular}

Spend income on household expenditure

\begin{tabular}{|c|c|c|c|c|c|c|c|}
\hline BPS 18 & $40(66.7)$ & $1(1.7)$ & $\begin{array}{c}19 \\
(31.7) \\
\end{array}$ & & & \multirow{5}{*}{$\begin{array}{l}0.02 \\
6\end{array}$} & \\
\hline BPS 19 & $24(85.7)$ & $2(7.1)$ & $2(7.1)$ & & & & 0.19 \\
\hline BPS 20 & 13(68.4) & $0(0.0)$ & $\begin{array}{c}6 \\
(31.6) \\
\end{array}$ & $\begin{array}{l}14.35 \\
5 \\
\end{array}$ & 6 & & \\
\hline BPS 21 & $\begin{array}{c}13(100 . \\
0)\end{array}$ & $0(0.0)$ & $0(0.0)$ & & & & \\
\hline Total & $\begin{array}{c}90 \\
(75.0)\end{array}$ & $3(2.5)$ & $\begin{array}{c}27 \\
(22.5)\end{array}$ & & & & \\
\hline
\end{tabular}

Independently scale or purchase property

\begin{tabular}{|c|c|c|c|c|c|c|}
\hline BPS 18 & $\begin{array}{c}21 \\
(35.0)\end{array}$ & $2(3.3)$ & $\begin{array}{c}37 \\
(61.7)\end{array}$ & & & \multirow{5}{*}{$\begin{array}{l}0.02 \\
5\end{array}$} \\
\hline BPS 19 & $\begin{array}{c}12 \\
(42.9)\end{array}$ & $5(17.9)$ & $\begin{array}{c}11 \\
(39.3)\end{array}$ & & & \\
\hline BPS 20 & $\begin{array}{c}11 \\
(57.9)\end{array}$ & $0(0.0)$ & $\begin{array}{c}8 \\
(42.1)\end{array}$ & $\begin{array}{l}14.50 \\
2\end{array}$ & 6 & \\
\hline BPS 21 & $4(30.8)$ & $0(0.0)$ & $\begin{array}{c}9 \\
(69.2)\end{array}$ & & & \\
\hline Total & $\begin{array}{c}48 \\
(40.0)\end{array}$ & $7(5.8)$ & $\begin{array}{c}65 \\
(54.2) \\
\end{array}$ & & & \\
\hline
\end{tabular}

\section{RESULT}

Respondents from all BPS are independent to spend their income on house hold expenditure.100.0\% from BPS 21, 85.7\% from BPS 19, 
$68.4 \%$ from BPS 20, 66.7\% from the BPS 18 are fully authoritative to spend their income for house hold items. There is significant association between the two attributes (Chi-square $=14.355 ; \mathrm{df}=6 ; \mathrm{p}=0.026$ ). women autonomy to purchase household items is positively correlated $(\mathrm{r}=.190)$ with their personal income, Decision regarding purchase OR scale property is significantly associated with pay scale of respondent (Chisquare $=14.502 ; \mathrm{df}=6 ; \mathrm{p}=0.025) .69 .2 \%(9 / 13)$ respondents from BPS 21 and $61.7 \%(37 / 60)$ cannot independently purchase OR scale property independently. On the other hand, 30.8\% (4/13) from the BPS 21 and $35.0 \%(21 / 60)$ from the BPS 18 have autonomy regarding property.

\section{HUSBAND'S POSSESSION OVER INCOME OF WIFE}

\begin{tabular}{|c|c|c|c|c|c|c|c|}
\hline $\begin{array}{c}\text { Respon } \\
\text { dent } \\
\text { basic } \\
\text { pay } \\
\text { scale } \\
\end{array}$ & Agree & $\begin{array}{c}\text { Undeci } \\
\text { ded }\end{array}$ & $\begin{array}{c}\text { Disagr } \\
\text { ee }\end{array}$ & $\begin{array}{c}\text { Chi } \\
- \\
\text { squ } \\
\text { are }\end{array}$ & $\begin{array}{l}\text { d } \\
\text { f }\end{array}$ & $\begin{array}{c}\text { p- } \\
\text { valu } \\
\text { e }\end{array}$ & $\begin{array}{c}\text { r- } \\
\text { value }\end{array}$ \\
\hline \multicolumn{8}{|c|}{ Spending income according to husband desire } \\
\hline BPS 18 & $\begin{array}{c}30 \\
(50.0)\end{array}$ & ------- & $\begin{array}{c}30 \\
(50.0)\end{array}$ & & & \multirow{5}{*}{$\begin{array}{c}0.04 \\
7\end{array}$} & \multirow{5}{*}{0.238} \\
\hline BPS 19 & $\begin{array}{c}14 \\
(50.0)\end{array}$ & 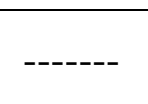 & $\begin{array}{c}14 \\
(50.0)\end{array}$ & & & & \\
\hline BPS 20 & $\begin{array}{c}5 \\
(26.3)\end{array}$ & -- & $\begin{array}{c}14 \\
(50.0)\end{array}$ & $\begin{array}{c}7.97 \\
3\end{array}$ & 3 & & \\
\hline BPS 21 & $\begin{array}{c}2 \\
(15.4) \\
\end{array}$ & & $\begin{array}{c}11 \\
(84.6) \\
\end{array}$ & & & & \\
\hline Total & $\begin{array}{c}51 \\
(42.2) \\
\end{array}$ & -- & $\begin{array}{c}69 \\
(57.5) \\
\end{array}$ & & & & \\
\hline \multicolumn{8}{|c|}{ Given complete income to husband } \\
\hline BPS 18 & $\begin{array}{c}14 \\
(23.3)\end{array}$ & $0(0.0)$ & $\begin{array}{c}46 \\
(76.6)\end{array}$ & & & \multirow{4}{*}{$\begin{array}{c}0.00 \\
8\end{array}$} & \multirow[t]{4}{*}{0.165} \\
\hline BPS 19 & $\begin{array}{c}11 \\
(39.3)\end{array}$ & $2(7.1)$ & $\begin{array}{c}15 \\
(53.6)\end{array}$ & & & & \\
\hline BPS 20 & $\begin{array}{c}2 \\
(10.5)\end{array}$ & $0(0.0)$ & $\begin{array}{c}17 \\
(89.5)\end{array}$ & $\begin{array}{c}17.5 \\
35\end{array}$ & 6 & & \\
\hline BPS 21 & $0(0.0)$ & $0(0.0)$ & 13 & & & & \\
\hline
\end{tabular}




\begin{tabular}{|c|c|c|c|c|c|c|c|}
\hline & & & $(100.0)$ & & & & \\
\hline Total & $\begin{array}{c}27 \\
(22.5) \\
\end{array}$ & $2(1.7)$ & $\begin{array}{c}91 \\
(75.8)\end{array}$ & & & & \\
\hline \multicolumn{8}{|c|}{ Husband manage all economic affairs of family } \\
\hline BPS 18 & $\begin{array}{c}37 \\
(61.7)\end{array}$ & $0(0.0)$ & $\begin{array}{c}23 \\
(38.3)\end{array}$ & & & \multirow{5}{*}{$\begin{array}{c}0.00 \\
0\end{array}$} & \multirow{5}{*}{0.401} \\
\hline BPS 19 & $\begin{array}{c}8 \\
(28.6)\end{array}$ & $2(7.1)$ & $\begin{array}{c}18 \\
(64.3)\end{array}$ & & & & \\
\hline BPS 20 & $\begin{array}{c}6 \\
(31.6)\end{array}$ & $0(0.0)$ & $\begin{array}{c}13 \\
(68.4)\end{array}$ & $\begin{array}{c}27.8 \\
25\end{array}$ & 6 & & \\
\hline BPS 21 & $0(0.0)$ & $0(0.0)$ & $\begin{array}{c}13 \\
(100.0)\end{array}$ & & & & \\
\hline Total & $\begin{array}{r}51 \\
(42.5) \\
\end{array}$ & $2(1.7)$ & $\begin{array}{c}67 \\
(55.8) \\
\end{array}$ & & & & \\
\hline \multicolumn{8}{|c|}{ Joint account with husband } \\
\hline BPS 18 & $\begin{array}{r}20 \\
(33.3)\end{array}$ & $\begin{array}{c}------- \\
-\end{array}$ & $\begin{array}{r}40 \\
(66.7)\end{array}$ & & & \multirow{5}{*}{$\begin{array}{c}0.04 \\
5\end{array}$} & \multirow{5}{*}{0.142} \\
\hline BPS 19 & $2(7.1)$ & $\begin{array}{c}------- \\
-\end{array}$ & $\begin{array}{r}26 \\
(92.9)\end{array}$ & & & & \\
\hline BPS 20 & $\begin{array}{r}3 \\
(15.8) \\
\end{array}$ & $\begin{array}{c}------- \\
-\end{array}$ & $\begin{array}{r}16 \\
(84.2)\end{array}$ & $\begin{array}{c}8.06 \\
2\end{array}$ & 6 & & \\
\hline BPS 21 & $\begin{array}{r}3 \\
(23.1)\end{array}$ & $\begin{array}{c}-------- \\
-\end{array}$ & $\begin{array}{r}10 \\
(76.9)\end{array}$ & & & & \\
\hline Total & $\begin{array}{r}28 \\
(23.3)\end{array}$ & $\begin{array}{c}------ \\
-\end{array}$ & $\begin{array}{r}92 \\
(76.7)\end{array}$ & & & & \\
\hline
\end{tabular}

\section{RESULT}

Result indicates significant association between (Chisquare $=14.502 ; \mathrm{df}=6 ; \mathrm{p}=0.047$ ) the two features. $50.0 \%$ from the BPS 18 and 50.0\% from the BPS 19 spend their income as their husband want, in contrast $84.6 \%(11 / 13)$ in BPS 21 and $73.7 \%$ (14/19) have autonomy on their income. It has strongly correlated $(\mathrm{r}=.238)$ with women decision making power. The entire respondent in BPS 21 has autonomy on their income. $89.5 \%$ from the BPS 20,76.6\% from the BPS 18, and $53.6 \%$ from 


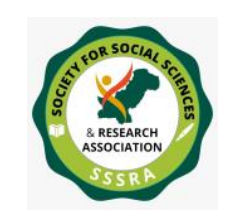

Pak. Journal of Int'L Affairs, Vol 1, Issue 2 (2018)

Study of Working - Women ,...

the BPS 19 don't give income to their husbands', on the other hand $39.3 \%(11 / 28)$ respondents from the BPS 19, 23.3\% (14/60) respondents from the BPS 18 and $10.5 \%(2 / 17)$ hand over their own personal income to husbands. The chi -square value is significantly associated (Chisquare $=17.535 ; \mathrm{df}=6 ; \mathrm{p}=0.008)$ in the mentioned distinctiveness positive correlation also observed $(\mathrm{r}=.165)$, Decision regarding management of economic affairs of house hold of respondents husband demonstrate significant association (Chi-square $=27.825 ; \mathrm{df}=6 \mathrm{p}=0.000$ ), $100.0 \%$ deny that decision regarding management of economic affairs of family is not handling by husband only, women dependency in economic affairs is strongly correlated $(\mathrm{r}=.401)$ management of economic affairs by husband. $68.4 \%, 64.3 \%, 38.3 \%$ from the BPS 20, BPS 19, and BPS 18 respectively refuse it, majority of the respondents do not in the favor of joint account with husband, Decision regarding joint account with husband is significantly associated basic pay scale of respondents (Chi-square=8.062; $\mathrm{df}=6 ; \mathrm{p}=0.045$ ). Separate account is correlated ( $\mathrm{r}=.142)$ with decision making power of married university teachers.

\section{DISCUSSION}

\section{AGE}

Autonomy of women in family matters is depends upon their age, level of education and their employment. Momentous impact of women age as a wife and as mother on decision making has been observed at family level (Casique,2002). Study demonstrate that the share of female involvement in family matters like child education, marriages, family planning, mobility etc lift as their age increase (Furuta, M., \& Salway, S, 2006) and Wives' involvement in family decision making process shows women talent to articulate their views additionally due to this they can influence the decisions of their male counterpart. Study demonstrate that women are more autonomous to take decisions as a mother (Casique, I, 2001) that is due to mother of living children more over with the passage of time as women get older their involvement in family related decisions also increased as a result they became more autonomous at family level ( Senarath U , \& Gunawardena , N.S, 2009). Seniority of husband in age also influence their decision-making power in our society husband are generally older than their wives and by the virtue of patriarchal society they became dominant over their wives (Adomako Ampofo A. , 1999), (Gyekye, 1996); Igboin, 2011.)

\section{PERSONAL INCOME / SOCIO - ECONOMIC STATUS}


Many changes occurred in the status of women in Pakistan. She is not only a house wife, but she is entered in the work force and prove herself a responsible member of her family by actively indulging in decision making process and attaing higher status but participation in labor force demand a lot from working women. Current study expose that role of income is significantly associated with decision making power of women and change occurred in their socio- economic status both in the household and societal level more over changes also observed in the attitudes of their husbands' they gave them more respect and gave them a chance to participate in the decision making process (Goetz, A. Mand, G.R.Sen 1996). To understand the decision-making power of women at household level researcher try to find out the link between the decisionmaking power of women and their demographic profile like their status, education, family monthly income, husband education, profession and residence etc. (Kishor ,2005). Researcher also make try to made association women archived status and their involvement in family matters. It is observed that status of female is determined by the level of education, income but authority or supremacy in decision making process depend upon their financial contribution, women who earn more than their male counterpart have more influence in decision making process (Sangsore and MC Granaha, 2003). Change is also observed in male counterpart attitudes, their behavior toward wives that enabling women to enjoy an independent economic life (Spitaze and Huber 1980; Thornton and freedmen, 1970).so it is concluded that economic status of women is significantly associated with their decision-making power (Agarwal, B, 1994).

\section{HOUSEHOLD EXPENDITURE / LESS AUTHORITY OF WOMEN OVER PROPERTY RELATED DECISION}

To improve the socio-economic standard of family the income of women plays vary significant role, an employed women gave their full contribute to the household expenses and their financial contribution increase their involvement in decision making process ( Adomako Ampofo , 1999; ) further more due to economic independency they can take decisions about personal needs and daily household expenses ( Sethi ,1998 ) not only this financial contribution also improve the status and position of women at household level that enhanced their decision making power (Agyeman, D. K., \& Casterline, J. B. , 2002) (Apusigah, A, 2009 ).Study reveals that women from all BPS Including BPS 18, BPS 19, BPS 20 , BPS 21 respectively spend their income on household expenses but they have less autonomy or right to take decisions concerning sale or 


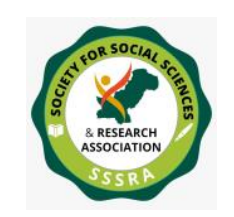

Pak. Journal of Int'L Affairs, Vol 1, Issue 2 (2018)

Study of Working - Women ,...

purchase property independently it is the matter of husband to take decisions a v.aa..zout assets like shelter land purchase of property (AngelUrdinola, 2010) resent study demonstrate the less authority of women over the economic decisions like ownership of property and land related issues (Arun, S., 1999) Due to education and employment women became aware about property right. However, in a modern senior husband with modern educated and employed wife take fewer decisions as compare to traditional husband (Green, R. T., \& Cunningham, I. C., 1975) (Madill, J., \& Bailey, S., 1999). It is observed that respondents' cultural background decides their level of domination over the issues related property (Hoyer WD and Maclnnis, DJ, 2004; Harcar. T, and Spillan, JE, 2006; Frikka, 2010). Moreover, it is find out that husband and wife take mutual decisions related property (Das, M.S, Lenna,1993).

\section{HUSBAND AUTONOMY OVER INCOME OF WIFE}

In a patriarchal social set up more specifically in( Quetta) Pakistan husband exercise more power over the economic resources of their family this un equal distribution of power effect the decision making power $f$ women such as less autonomy over their own income, less control over financial resources, this is because of supreme position of males as a head of family member either in ( Nuclear, joint) family manage all economic affairs of their family so women due to their submissive attitudes plays vary little role in decision making (Wrigley-Asante, C., 2008) although women employment and income play vary significant role to achieve the decision making power both in family level as well as societal level too but in patriarchal social setting elder male member or husband manage all economic affairs of their family, so how to manage the economic matters of family is the concern of husband but decisions related children education and marriages spouses often consult each other furthermore the decisions of women considered only ,unimportant matters like choosing the household decoration items (Edgell, S. R. 1980. , 1980)But highly educated women have self-assurance or confidence of discussion for access towards resources (Malhotra, A., \& Mather, M. , 1997).

\section{CONCLUSION}

A woman is not only the best creature of the world but also the integral part of any society. The progress of any family or society is not possible without the active participation of the women in any sphere of the life. Due to industrial revolution women became independent also became aware about their rights that is showed by many researches but in Quetta 
Baluchistan the women decision-making power is limited because of patriarchal social set up, although she has an ability to influence others by their ideas, knowledge and thoughts. She performs many tasks under the supervision of their men. In this context the present study explored the factors that may influence the decision-making patterns of husband and wives in family. The result showed that women education and employment play significant role to make them empowered in the process of decision making at family level. Due to this she became able to achieve the socio-economic status in society. Study also reveals age as a significant factor influencing decision making power of University teachers changes are observed in the decision-making power of married women belonged to the age group 30-40 have more autonomy to take decisions regarding house hold matters like spend income on household expenditures but they have no right to sale or parches any kind of property by their own as compare to those working women who belong to the age group of 50 and above

\section{SUGGESTIONS}

1. Equal participation should be encouraging to improve the standard of family which further help to develop the society in a smooth way

2. For the social economic development of the country involvement of educated women should be considered at family level as well as social and political point of view

\section{LIMITATION AND FRAME WORK FOR FURTHER RESEARCH}

1. Decision making is a complex phenomenon researcher through quantitative analysis try to find out the factors influencing the decision-making power of married women which provide a lack of in depth information which may sort out by qualitative research design.

2. Study is limited universities teachers to develop a considerate policies sample size should be included from all fields of life

3. This study is confined only to university teachers that can be extended to all type of working women of Public and Private sectors.

4. Present study is based on role of age and income in decision making further studies can be done on family system 


\section{REFERENCES}

Arooj,S,Hussain.(2013).Paid work and decision making power of married women cross sectional survey of Muzaffarabad Azad state of Jammu\& Kashmir. Advances in Applied Sociology, 3(03)165.

Adomako, Ampofo, A. (1999). Resource contributions, gender orientation, and reproductive decision making in Ghana;(The case of urban couples). Research Review 2(15), 93-125.

Amanor-Wilks, D.E. (2009). Land and labour and gendered livelihood in a peasant, and a settler economy. Feminist Africa, 12,31-50.

Angel-Urdinola, D. (2010). Income generation and intera household decision making: A gender analysis for Nigeria. Gender Disparities in Africa's Labor Market, 381.

Agarwal, B. (1994). Gender and command over property: Acritical gap in economic analysis and policy in south Asia. World development, 22(10), 1455-1478.

Arun, S. (1999). Does land ownership make a difference? Women's role in agriculture in Kerala, India. Gender and Development, 7 (3), 19-27.

Apusigah, A. (2009). The gendered politics of farm household production and the shaping of women's livelihoods in northen Ghana. Land, Labour and Gendered Livelihood. Feminist Africa, 51-68.

Carlsson, F.M. (2009). Household decision making and the influence of spouses' income, education, and communist party membership:Afield experiment in china.

Casique, M. (2000). Mexican married women's autonomy and power within the household. Latin Amercian Studies Association, Rio de Janeiro, January.

Casique, I, (2001). Women autonomy and power and use of contraception in Mexico: What difference does it make. In Salvador, XXIV IUSSP General Population Conference, 18-24. Brazil. 
Das, M.S, Lenna. (1993). Decision making practice of employed and nonemployed home makers. Journal of Northeast India Council for Social Sciences Research, 17, 46-78.

Dutta, M. (2002). Wome's power and authority with in middle class hold in Kolkata. Contemporary South Asia .11(1),7-18.

Denise, B. Kandel and Gerald S. Lesser. (1972). Marital Decision making in American and Danish urban families: A Research Note. Journal of marriage and family., 34(1):134-138.

Edgell, S. R. (1980). Middle class couples. London. International journal of sociology of family 12 (1), 135-135.

Fatima, D. (2013) Education, Employment and women' say in house hold decision making. In Pakistan. Diss. @ Lahore School of Economics.

Frikka A. (2010). Conflict in purchase decision making with in couples. Journal of Islamic Marketing. 1 (3), 231-248.

Furuta, M., \&Salway, S. (2006). Women position within the household as a determinant of meternal health care use in Nepal. International Family Planning Perspective, 17-27.

Green R, Cunningham. (1975) Feminine Role Perception and Family Purchasing Decision. Journal of Marketing Research 12, 325-332.

Gyekye, K. (1996). African cultural values: An introduction. Accra: Sankofa publishing company.

Goetz, A. M and, G.R.Sen. (1996). Who takes credit? Gender power and control over loan use in rural credit programs in Bangladesh. World Development ,24(1),45-63.

Hakim abdul and Aziz azra .(1998).Socio cultural ,religious and political aspects of the status of women in Pakistan . The Pakistan Development Review ,37(4),727-746.

Hammad, M. (2010), THhe Effect of Women's Work Outside the House On Family Decision Making in Bethlehem. Available at SSRN 1669096. 
Harcar, T, and Spillan J.E. (2006). Exploring Latin American family decision-making using correspondence analysis. Journal of World Business, 41, 221-232.

Hoyer WD, Macinnis DJ. (2004). Consumer behavior, (3 ${ }^{\text {rd }}$ ed). Boston: Houghton Mifflin Company.

Jabeen, N. and Iqbal. M.Z. (2010). Gender and Local Goverance in Pakistan:Promoting Participation through Capacity Building, South Asian Studies 25(2),255

Jahan, F, Hossain,S,Mahmud, K.M.F.(2015).Factors Influencing Women's Decision Making Power:Evidence From Bangladesh Urban Health Survey Data. International Journal of Research in Applied Natural and Social Sciences,3(3),133-150.

Jan, M, \& Akhter, S. (2008). An analysis of decision making power among married and un married women. Kamal- Raj Stud, Home Comm. Sci,2(1),43-50

Kandel, D.b(1972). Marital Decision Making in American and Danish Urban Families. Journal of Marriage and Family,34(1),134-138

Khanam, R. (2003). Family Decision -Making Pattern of Husband and Wife: an urban case study in Bangladesh.

Kishor,S,\&\& Gupta,K.(2004). Women's empowerment in India and its states:evidence from the NFHS. Economic and Political Weekly, 39(7), 694-712.

Lee.C,K.Beatty,S.E.(2002) Family structure and early child hood mortality in Bangladesh. Journal of Consumer Marketing 19(1), 24-41.

Madill, JJ, \&Bailey,S.( 1999) Household decision making: The relative influence of husband and wives in the 1990s. European Advanced in Consumer Research, 4, 232-237.

Malhotra, A., \& Mather, M. (1997). Do schooling and work empower women in developing countries? Gender and domestic decisions in Sri Lanka. In sociological Form, Kluwer Academic Publishers, 12(4), 599630. 
Muzamil, J and A. shubeena. (2008). An analysis of decision making power among married and unmarried women, stud. Home Comm.Sci.,2(1):43-50

Rizvi, P. (1980). Babkground of vocational education for girls. The national conference on critical issues concering women in education, Govt of Pakistan, Islamabad.

Safilios- Rothschild,C. (1969). Family sociology. A cross cultural examination of decision making. Journal of Marriage and the Family, 290-301.

Sultana, A. (2010). Patriarchy and women's Gender Ideology: A Socio cultural perspective. Journal of Social Sciences., 6(1), 123-126.

Senarath U, Gunawardena. N.S. (2009). Women's autonomy in decision making for health care in south Asia. Asia- Pacific Journal of Public Health. 21, 137-143.

Sethi.R.M.(1998). Status and power of working women with in family. Journal of Sociological Studies. 8, 97-108.

Songsore,J and G. Mc Granahan. (2003). Women's household environmental caring roles in the greater Accra Metropolitan Area: A Qualitative Appraisal. Institute of African Studies Research Review. University of Ghana, Legon, 19(2), 67-83.

Spitze, G., \& Huber, J. (1980). Changing attitude towards women's nonfamily roles 1938 to1978. Work and Occupations, 7(3),317-335.

Sultana, A. (2010). Patriarchy and women's gender ideology:A socioCultural Perspective.Journal of Social Sciences., 6(!),123-126

Taj, S.A. (2004). Assessment of rural and urban women's participation in the decision making in family matters, Pak. J. life and S. Sci., 2(1), 28-32

Thornton, A., \& Freedman, D. (1979). Changes in the sex role attitudes of women, 1962-1977: Evidence from a panel study. American Sociological Review, 831-842. 
Wrigley- Asante, C. (2008). Men are poor but women are poorer: Gendered poverty and survival strategies in the Dangme District og Ghana. Norsk Geografisk Tidsskrift- Norwegian Journal of Geography, $62(3), 161-170$. 